\title{
Incomplete Tests for Undetectable Faults to Improve Test Set Quality
}

\author{
IRITH POMERANZ, Purdue University
}

The presence of undetectable faults in a set of target faults implies that tests, which may be important for detecting defects, are missing from the test set. This article suggests an approach for addressing missing tests that fits with the rationale for computing an $n$-detection test set. The artcile defines the concept of an incomplete test that is relevant when a target fault is undetectable. An incomplete test activates the fault but fails to detect it because of one or more assignments that are missing from the test. The procedure described in this article improves the quality of a test set by attempting to ensure that every undetectable fault has $n$ incomplete tests with the smallest possible numbers of missing assignments, for a constant $n \geq 1$. The incomplete tests are expected to contribute to the detection of detectable defects around the site of the undetectable fault. The computation of missing assignments for a test is performed in linear time by avoiding fault simulation and considering all the undetectable faults simultaneously. Experimental results demonstrate the extent to which a given test set can be improved without increasing the number of tests.

\section{CCS Concepts: • Hardware $\rightarrow$ Test-pattern generation and fault simulation;}

Additional Key Words and Phrases: $n$-detection test set, test generation, test set quality, undetectable faults

ACM Reference format:

Irith Pomeranz. 2019. Incomplete Tests for Undetectable Faults to Improve Test Set Quality. ACM Trans. Des. Autom. Electron. Syst. 24, 2, Article 23 (February 2019), 13 pages.

https://doi.org/10.1145/3306493

\section{INTRODUCTION}

A high-quality test set can be generated by targeting faults from more than one fault model. For example, a test set can target the detection of bridging [1-7] and open [8-16] faults. The fault models can be matched to the expected behaviors of defects in a specific technology [17-20]. An understanding of the causes for defects is also important for the definition of cell-aware faults [21-24].

Gate-exhaustive [25-29] and $n$-detection [30-35] test generation procedures attempt to address the fact that not all the possible defects are known in advance and understood well enough to select fault models for them. To ensure that the test set covers unexpected defects, a gate-exhaustive

This work was supported in part by NSF Grant No. CCF-1714147.

Author's address: I. Pomeranz, School of Electrical and Computer Engineering, Purdue University, West Lafayette, IN 47907, USA; email: pomeranz@ecn.purdue.edu.

Permission to make digital or hard copies of all or part of this work for personal or classroom use is granted without fee provided that copies are not made or distributed for profit or commercial advantage and that copies bear this notice and the full citation on the first page. Copyrights for components of this work owned by others than ACM must be honored. Abstracting with credit is permitted. To copy otherwise, or republish, to post on servers or to redistribute to lists, requires prior specific permission and/or a fee. Request permissions from permissions@acm.org.

(C) 2019 Association for Computing Machinery.

1084-4309/2019/02-ART23 \$15.00

https://doi.org/10.1145/3306493 


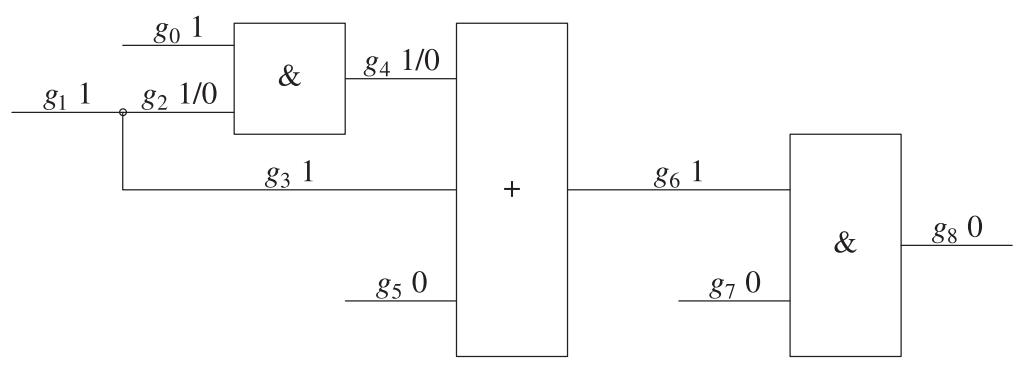

Fig. 1. Example of an incomplete test.

approach tests gates exhaustively, and an $n$-detection test set includes $n$ different tests for every target fault. The tests create different conditions around a gate or fault site, thus contributing to the detection of defects in these areas. This is expected to compensate for the possibility that defect behavior is not captured accurately by a fault model.

Considering specific fault models, the procedures described in References [36, 37] address the presence of undetectable faults based on the following observation. Let $f$ be a target fault. A test for $f$ is expected to detect the fault $f$ if it occurs in the circuit, as well as defects around the site of $f$. If $f$ is undetectable, then a test that would have detected it is missing from the test set. As a result, the test set may fail to detect defects around the site of $f$, even if these defects are detectable. The issue of a missing test for an undetectable fault $f$ is addressed in References [36, 37] by adding to the test set tests for different faults around the site of $f$. In Reference [36], a bridging fault model is defined to address the sites of undetectable transition faults. In Reference [37], what are called unspecified transition faults are used for addressing undetectable transition faults under multicycle tests.

To remove the reliance that exists in References [36,37] on specific fault models, it is possible to use gate-exhaustive faults to cover the sites of undetectable target faults. This article takes a more general approach that fits with the rationale for computing an $n$-detection test set. The article defines the concept of an incomplete test that is relevant when a target fault is undetectable. An incomplete test activates the fault but fails to detect it because of one or more assignments that are missing from the test.

An example is shown in Figure 1, where the fault $g_{2}$ stuck-at 0 is undetectable. The test shown in Figure 1 activates the fault by assigning the value 1 to line $g_{2}$ in the fault-free circuit. The test also propagates the fault effect $1 / 0$ to line $g_{4}$. However, the value one on $g_{3}$ prevents the fault effect from propagating further. The value $g_{5}=0$ would have allowed the fault effect to propagate, but $g_{7}=0$ would have stopped its propagation. The test is referred to as an incomplete test with two missing assignments, where the missing assignments are caused by $g_{3}=1$ and $g_{7}=0$.

The procedure described in this article improves the quality of a test set by attempting to ensure that every undetectable fault has $n$ incomplete tests with the smallest possible numbers of missing assignments, for a constant $n \geq 1$. The incomplete tests are expected to contribute to the detection of detectable defects around the site of the undetectable fault. Such defects are expected to require propagation paths that are similar to the ones required for the undetectable fault. Therefore, even missing assignments that are far from the fault site are important. The importance of a small number of missing assignments is that it makes it more likely that detectable defects will be detected by an incomplete test. Specifically, with a smaller number of missing assignments, it is more likely that a propagation path will exist for a detectable defect. The main challenge in the computation of missing assignments for a test is to avoid repeated fault simulation to determine which assignments are missing. The procedure described in this article addresses this 
challenge by counting the number of missing assignments based only on fault-free values and traversing the circuit three times for this purpose. This results in a computational complexity that is linear in the number of lines in the circuit for computing the missing assignments of a test.

The procedure is designed such that it does not increase the number of tests in the test set given to it. This approach is used by the $n$-detection test generation procedure from Reference [34]. It is suitable here, because incomplete tests do not detect known faults. In contrast, in References [36, 37], as well as when gate-exhaustive faults are considered, tests are added to the test set to detect known faults.

Only single stuck-at faults are considered in this article. The concepts of missing assignments and incomplete tests can be applied to other fault models.

The article is organized as follows. The procedure for counting the number of missing assignments for a test is described in Section 2. Section 3 describes the procedure for improving a given test set by increasing the numbers of incomplete tests for undetectable faults and reducing the numbers of missing assignments for these tests. Section 4 presents experimental results.

\section{COUNTING MISSING ASSIGNMENTS}

Let $F$ be the set of target faults. Let the faults in $U \subseteq F$ be undetectable. This section considers a test $t$ as an incomplete test for the faults in $U$. For every fault $f \in U$, the procedure described in this section computes the number of missing assignments under $t$. This number is denoted by $\mu(f, t)$. This section also defines parameters related to the numbers of missing assignments under a test set $T$.

The procedure requires three traversals of the circuit. Each traversal has a computational complexity that is linear in the number of lines in the circuit. In the first traversal, the procedure performs logic simulation of $t$ to find a value $v(g)$ for every line $g$. In the second traversal, the procedure performs a local analysis of missing assignments. In the third traversal, the procedure performs a global analysis of missing assignments that considers paths for propagating fault effects.

The second and third traversals are described next.

\subsection{Local Missing Assignments}

In the second traversal, the procedure computes a number of missing assignments $\mu_{l c l}(g)$ locally for every line $g$. Initially, $\mu_{l c l}(g)=0$ for every line $g$. If $g$ is not a gate input, then $\mu_{l c l}(g)=0$ remains. Suppose that $g$ is the input of a gate with a controlling value $c$, and inputs $g, g_{0}, g_{1}, \ldots, g_{k-2}$. For $0 \leq i<k-1$, the procedure adds one to $\mu_{l c l}(g)$ for every gate input $g_{i}$ such that $g_{i}=c$. In this way, the procedure counts in $\mu_{l c l}(g)$ the number of assignments to other inputs of the same gate that prevent the propagation of fault effects from $g$.

For illustration, Figure 2 shows the values of $\mu_{l c l}(g)$ for the circuit and test from Figure 1. For every line $g$, the values are shown in the format $v(g), \mu_{l c l}(g)$. For example, $\mu_{l c l}\left(g_{2}\right)=0$ because of the non-controlling value on line $g_{0} ; \mu_{l c l}\left(g_{4}\right)=1$ because of the controlling value on line $g_{3}$, and the non-controlling value on line $g_{5}$; and $\mu_{l c l}\left(g_{5}\right)=2$ because of the controlling values on lines $g_{3}$ and $g_{4}$.

The computation of local missing assignments can be performed in linear time as follows. Every gate output is considered separately. For every gate output, the number of controlling input values is counted, and assigned to a variable denoted by $n_{c}$. For a gate input $g$ with a non-controlling value, $\mu_{l c l}(g)=n_{c}$. For a gate input $g$ with a controlling value, $\mu_{l c l}(g)=n_{c}-1$. Since every line is considered at most twice, once for counting $n_{c}$ and once for assigning $\mu_{l c l}(g)$, the computational complexity is linear in the number of lines. 


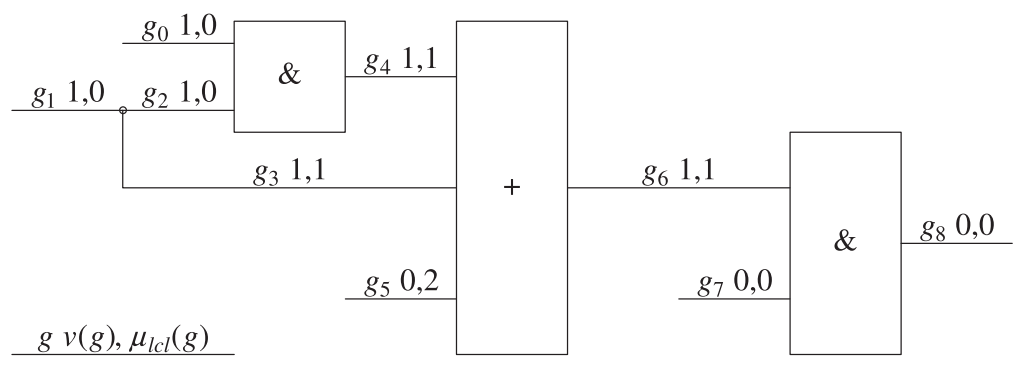

Fig. 2. Example of local missing assignments.

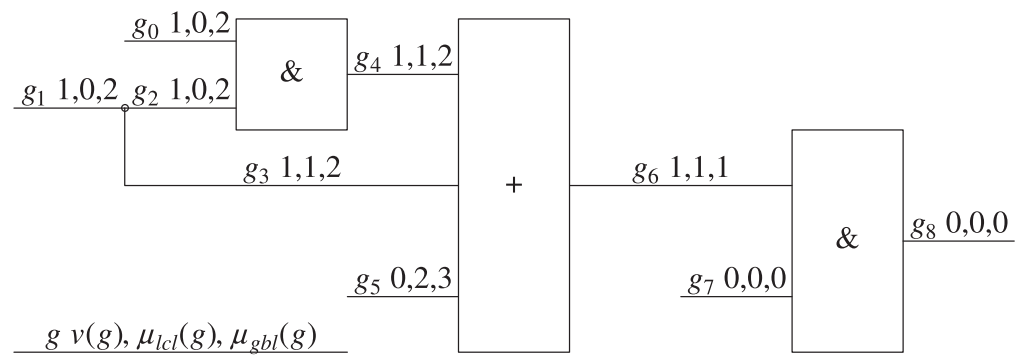

Fig. 3. Example of global missing assignments.

\subsection{Global Missing Assignments}

The third traversal of the circuit computes a number of missing assignments $\mu_{g l b}(g)$ for every line $g$ taking into consideration that fault effects need to propagate through a path from $g$ to an output. The computation proceeds from the outputs to the inputs. It results in the minimum number of missing assignments on a path from $g$ to an output under the given test. The computation uses the numbers of missing assignments $\mu_{l c l}(g)$ that were computed locally. The computation is not accurate, because it does not take into consideration interactions between values, and the possibility of propagating fault effects through multiple paths. However, accuracy is not necessary, since the goal is not to generate a test that detects detectable target faults, but to obtain a metric for how far a test is from detecting an undetectable target fault. A test with fewer missing assignments is expected to be better, because it is more likely to detect detectable defects. The advantage of the metric as it is defined here is that it can be computed in linear time, and thus can be used as part of a test generation procedure that computes incomplete tests.

The third traversal initially assigns $\mu_{g l b}(g)=0$ for every output $g$ of the circuit, and $\mu_{g l b}(g)=\infty$ for every other line. When a line $g$ is considered in the order from outputs to inputs, the procedure makes the following updates. If $g$ is a gate output, then the procedure considers the gate inputs $g_{0}, g_{1}, \ldots, g_{k-1}$. If $g$ is a fanout branch, then the stem $g_{0}$ is considered its only input, and $k=1$. For $0 \leq i<k$, the procedure compares the current value of $\mu_{g l b}\left(g_{i}\right)$ with the value that would be assigned to it by considering a propagation path that goes through $g$. The number of missing assignments for a propagation path through $g$ is equal to $\mu_{g l b}(g)+\mu_{l c l}\left(g_{i}\right)$, where $\mu_{g l b}(g)$ accounts for the path from $g$ to an output, and $\mu_{l c l}\left(g_{i}\right)$ accounts for the local missing assignment of $g_{i}$. If $\mu_{g l b}(g)+\mu_{l c l}\left(g_{i}\right)<\mu_{g l b}\left(g_{i}\right)$, then the path through $g$ is preferred, and the procedure assigns $\mu_{g l b}\left(g_{i}\right)=\mu_{g l b}(g)+\mu_{l c l}\left(g_{i}\right)$.

For illustration, Figure 3 shows the values of $\mu_{g l b}(g)$ for the circuit and test from Figure 2. For every line $g$, the values are shown in the format $v(g), \mu_{l c l}(g), \mu_{g l b}(g)$. Considering the fault $g_{2}$ stuck-at 0 from Figure 1, we have that $\mu_{g l b}\left(g_{2}\right)=2$. This matches the fact that the values of $g_{3}$ and 


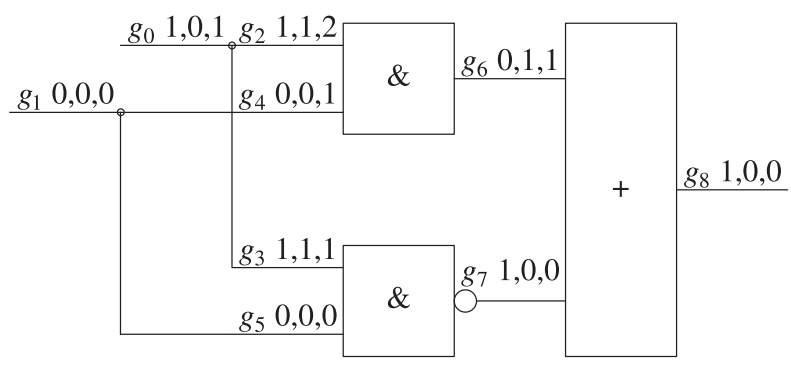

Fig. 4. Example without missing assignments.

$g_{7}$ stop the propagation of the fault effect. The controlling value of $g_{7}$ is reflected in $\mu_{l c l}\left(g_{6}\right)$, and results in $\mu_{g l b}\left(g_{6}\right)=1$. The controlling value of $g_{3}$ is reflected in $\mu_{l c l}\left(g_{4}\right)$. With $\mu_{g l b}\left(g_{6}\right)=1$ and $\mu_{l c l}\left(g_{4}\right)=1$ the result is $\mu_{g l b}\left(g_{4}\right)=2$. With $\mu_{g l b}\left(g_{4}\right)=2$ and $\mu_{l c l}\left(g_{2}\right)=0$ the result is $\mu_{g l b}\left(g_{2}\right)=2$.

Since every line is considered twice in this traversal, once as a gate output and once as a gate input, the computational complexity is linear in the number of lines.

\subsection{Missing Assignments for Undetectable Faults}

After the three traversals, for every fault $f \in U, \mu(f, t)$ is computed as follows. Let the fault $f$ be the fault $g$ stuck-at $a$. If $t$ assigns $g=a$, then it does not activate the fault. In this case, $\mu(f, t)=\infty$. If $t$ assigns $g=a^{\prime}$, then $\mu(f, t)=\mu_{g l b}(g)$.

Given a test set $T$ and a set of undetectable faults $U$, the subset of undetectable faults that are activated by $T$ is denoted by $A$. For a fault $f \in A$, every test $t \in T$ that activates $f$ yields a number $\mu(f, t)$ of missing assignments. Let $M(f)=\{\mu(f, t)<\infty: t \in T\}$. For a constant $n \geq 1$, if the number of tests that activate $f$ is larger than $n$, then the procedure keeps in $M(f)$ only the $n$ smallest values.

For example, suppose that a test set contains seven tests that activate a fault $f$, and $M(f)=$ $\{1,1,1,2,3,3,5\}$. With $n=4$, the procedure uses $M(f)=\{1,1,1,2\}$. We have that $|M(f)|=4$ and the average value of an entry of $M(f)$ is $\overline{M(f)}=1.25$.

Finally, it is interesting to note that the inaccuracy in the computation of the metric $\mu(f, t)$ can lead to a situation where $\mu(f, t)=0$ for an undetectable fault. An example to illustrate this possibility is shown in Figure 4. The fault under consideration is $g_{1}$ stuck-at 1 . The test in Figure 4 activates the fault, and creates a propagation path $g_{1}-g_{5}-g_{7}-g_{8}$ that results in $\mu(f, t)=0$. However, fault effects propagate to $g_{6}$ and $g_{7}$, and cancel each other. As a result, the fault is not detected. The fault is undetectable, since $g_{0}=1$ and $g_{1}=0$ are both necessary for activating and propagating the fault.

\section{PROCEDURE FOR TEST SET IMPROVEMENT}

This section describes a procedure that accepts a test set $T=\left\{t_{0}, t_{1}, \ldots, t_{K-1}\right\}$, which may be generated by any test generation procedure and target any number of detections. The procedure improves the quality of the test set as an $n$-detection test set, where $n \geq 1$, by: (1) preserving the numbers of detections for detectable faults, and (2) for undetectable faults, increasing the numbers of incomplete tests, and reducing their numbers of missing assignments.

The procedure does not increase the number of tests in the test set $T$ given to it. Instead, it modifies the tests that are already included in $T$ to achieve its objectives. In addition, the procedure does not attempt to increase the numbers of detections of detectable faults.

The procedure considers the tests in $T$ for modification one by one in the order $t_{K-1}, t_{K-2}, \ldots, t_{0}$. The premise is that the tests at the end of $T$ are easier to modify, since they were generated after more of the detectable faults have already been detected the required number of times. 
Table 1. Example of Test Modification

\begin{tabular}{c|ccccccccccccccc}
\hline & \multicolumn{10}{|c}{$\mu\left(f, t_{i}\right)$ for $f=$} \\
$j$ & $f_{180}$ & $f_{477}$ & $f_{575}$ & $f_{638}$ & $f_{717}$ & $f_{908}$ & $f_{983}$ & $f_{1008}$ & $f_{1023}$ & $f_{1028}$ & $f_{1217}$ & $f_{1336}$ & $f_{1435}$ & $f_{1437}$ \\
\hline- & - & 3 & 2 & 2 & 2 & - & - & 2 & - & 2 & - & - & - & - \\
0 & - & 3 & 2 & 2 & 2 & 6 & - & 2 & - & 2 & - & - & - & - \\
1 & - & 3 & 2 & 2 & 2 & 5 & - & 2 & - & 2 & - & - & - & - \\
2 & - & 3 & 2 & 2 & 2 & 3 & - & 2 & - & 2 & - & - & - & - \\
37 & 3 & 3 & 2 & 2 & 2 & 3 & - & 2 & - & 2 & - & - & - & - \\
81 & 3 & 3 & 2 & 1 & 2 & 3 & - & 2 & - & 2 & - & - & - & - \\
\hline
\end{tabular}

To modify a test $t_{i} \in T$, the procedure moves $t_{i}$ to the end of the test set. For the reordered test set, it performs $n$-detection fault simulation of $T$. For the undetectable faults in $U$, it computes the sets $M(f)$. The procedure considers the following two subsets of target faults for the modification. With $t_{i}$ at the end of $T$, fewer target faults are obtained, and it is easier to modify $t_{i}$.

A fault $f \in F-U$ that is detected by $t_{i}$ is included in a set of target faults $D_{i}$.

A fault $f \in U$ is included in a set of target faults $U_{i}$ if $|M(f)|<n$, or $\overline{M(f)}>1$, or $t_{i}$ contributes an entry to $M(f)$. In these cases, it is important for $t_{i}$ to be an incomplete test for $f$ with the smallest possible number of missing assignments. For the faults in $U_{i}$, the procedure records the numbers of missing assignments $\mu\left(f, t_{i}\right)$.

The procedure modifies $t_{i}$ by complementing its bits one at a time. After complementing bit $j$, let the test $t_{i}^{j}$ be obtained. The procedure decides whether or not to accept the complementation as follows.

It simulates $D_{i}$ under $t_{i}^{j}$. If any of the faults in $D_{i}$ is not detected, then the complementation is not accepted.

It computes $\mu\left(f, t_{i}^{j}\right)$ for every fault $f \in U_{i}$. If $\mu\left(f, t_{i}^{j}\right)>\mu\left(f, t_{i}\right)$ for any fault $f \in U_{i}$, then the complementation is not accepted.

If all the faults in $D_{i}$ are detected, and $\mu\left(f, t_{i}^{j}\right) \leq \mu\left(f, t_{i}\right)$ is obtained for every fault $f \in U_{i}$, then the complementation is accepted. In this case, the procedure assigns $t_{i}=t_{i}^{j}$.

An example is given in Table 1, where a test $t_{i}$ of benchmark circuit $s 1423$ is modified based on 14 undetectable stuck-at faults. The faults are shown as column headers. Column $j$ shows the bits whose complementation is accepted. The dash corresponds to the initial test, before any bits are complemented.

Table 1 shows the values of $\mu\left(f, t_{i}\right)$ for the initial test, and after the complementation of bit $j$ is accepted, for $j=0,1,2,37$ and 81 . A dash for $\mu\left(f, t_{i}\right)$ indicates that the fault is not activated. For example, fault $f_{908}$ is not activated by the initial test. It is activated by complementing bit $j=0$, with six missing assignments. The number of missing assignments is reduced to five after complementing bit $j=1$, and then to three after complementing bit $j=2$.

An improvement in the test set occurs after $t_{i}$ is modified if new faults are activated (the size of the set $A$ increases), or the number of activating tests for a fault in $U$ is increased $(|M(f)|$ increases for at least one fault $f \in A$ ), or the average number of missing assignments for an activating test is decreased $(\overline{M(f)}$ is decreased for at least one fault $f \in A)$.

The procedure is summarized next.

Procedure 1: Test set improvement

(1) For $i=K-1, K-2, \ldots, 0$ :

(a) Move $t_{i}$ to the end of the test set.

(b) Perform $n$-detection fault simulation. 
(c) Compute the sets $M(f)$ for $f \in U$.

(d) Assign $D_{i}=\left\{f \in F-U: f\right.$ is detected by $\left.t_{i}\right\}$.

(e) Assign $U_{i}=\left\{f \in U:|M(f)|<n\right.$ or $\overline{M(f)}>1$ or $t_{i}$ contributes an entry to $\left.M(f)\right\}$.

(f) For every bit $j$ of $t_{i}$ :

(i) Complement bit $j$ to obtain the test $t_{i}^{j}$.

(ii) Simulate $D_{i}$ under $t_{i}^{j}$.

(iii) Compute $\mu\left(f, t_{i}^{j}\right)$ for every fault $f \in U_{i}$.

(iv) If all the faults in $D_{i}$ are detected, and $\mu\left(f, t_{i}^{j}\right) \leq \mu\left(f, t_{i}\right)$ for every fault $f \in U_{i}$, then assign $t_{i}=t_{i}^{j}$.

The worst-case computational complexity of Procedure 1 is determined by the modification of the tests from $T$. Let the number of bits in a test be $B$. The procedure simulates $B$ modified tests for every test in $T$. The union of all the subsets of faults $D_{i}$ that the procedure simulates is at most $F$. This implies that the procedure performs fault simulation for $O(B|F|)$ tests and faults.

\section{EXPERIMENTAL RESULTS}

The results of the test set improvement procedure for single stuck-at faults in benchmark circuits are presented in this section.

\subsection{Setup}

The procedure is applied to compact one-detection and 10-detection test sets for single stuck-at faults. For further illustration, for several circuits, the procedure is also applied to a one-detection test set that is extended with tests for gate-exhaustive faults to improve the coverage of sites of undetectable faults.

Most of the test sets detect all the detectable faults. If not, then the undetected faults are included in the set $U$ together with the undetectable faults. While the procedure attempts to improve the test set, it may detect such a fault accidentally.

The target $n$ for the number of incomplete tests for an undetectable fault $f \in U$ is $n=16$. In addition, $n$-detection fault simulation for detectable faults is carried out using $n=16$. This allows the numbers of detections of target faults to be used as an independent quality metric. With $n=16$, the modification of the tests is expected to increase the numbers of detections of detectable faults even under the ten-detection test set.

\subsection{Results}

Tables 2 and 3 show the results of the test set improvement procedure. There are two rows for every circuit and test set. The first row describes the initial test set. The second row describes the final test set obtained by the test set improvement procedure. This test set is obtained after modifying the last test that results in an improvement of the test set.

For every test set, column pi shows the number of primary inputs. Column $t s$ shows whether the one-detection or 10-detection test set is considered. A one-detection test set that is extended with tests for gate-exhaustive faults has $1 G$ under column $t$. Column tests shows the number of tests in the test set. The number of tests does not change by the improvement procedure. Column mod shows the index of the modified test.

Column $f$.c. shows the fault coverage. Column 16det shows the average number of tests for a detectable fault when 16-detection fault simulation is carried out.

Column undet shows the number of undetectable faults. This is the size of the set $U$. Column act shows the number of undetectable faults that are activated by the test set. This is the size of 
the set $A$. Column targ shows the number of undetectable faults that are targeted by the test set improvement procedure. Column 16act shows the average number of incomplete tests for a fault in $A$. Column miss shows the average number of missing assignments, or the average value of $\overline{M(f)}$, considering all the faults in $A$.

Column ntime shows the normalized run time as follows. Let the run time for $n$-detection fault simulation of the initial test set be $\rho_{\text {base }}$. For the initial test set, the procedure also computes the sets $M(f)$ for the faults in $U$. Let the total run time for the initial test set be $\rho_{\text {init }}$. Let the total cumulative run time of the improvement procedure be $\rho_{\text {improv }}$. The normalized run time is $\rho_{\text {init }} / \rho_{\text {base }}$ in the first row, and $\rho_{\text {improv }} / \rho_{\text {base }}$ in the second row.

\subsection{Discussion}

The following points can be seen from Tables 2 and 3. In several cases, the test set improvement procedure increases the average number of incomplete tests for a fault in $A$. In most of the cases, it reduces the average number of missing assignments.

The improvement in the quality of the test set can be seen from these parameters, as well as the average number of detections of detectable faults. This number increases even though the procedure does not target it directly. The importance of increasing the numbers of detections of target faults to improve the ability of a test set to detect defects is discussed in References [30-35].

As may be expected, when the 1-detection test set is extended with tests for gate-exhaustive faults, or when the 10-detection test set is used, the test set has larger numbers of incomplete tests for undetectable faults, and fewer missing assignments. Nevertheless, the improvement procedure is able to improve these test sets as well.

The normalized run time for the initial test set shows that the computation of the numbers of missing assignments for the undetectable faults has a negligible contribution to the run time. This is important for making it possible to use this metric during test generation. Overall, the normalized runtime does not increase with the size of the circuit. This implies that the procedure scales similar to a fault simulation procedure. To further demonstrate this point, Figure 5 shows the normalized run time as a function of the number of primary inputs for the one-detection test set.

The normalized run time is higher for the 10-detection test set because of the larger number of tests that the procedure considers for modification. The normalized runtime is also higher when the last modified test is closer to the beginning of the test set, implying that more tests are considered for modification. This occurs, for example, for $b 14$ and $t v 80$.

\subsection{Bridging Faults}

As an additional metric for the quality of the test set, a bridging fault coverage is computed in this section. Bridging faults are not targeted by the test sets or procedure in this article. Therefore, bridging faults can be used for representing unmodeled faults, and defects.

The four-way bridging fault model is used in this section. A four-way bridging fault is associated with two lines, $g$ and $h$, and a value $a \in\{0,1\}$. In the presence of the fault, the value $a$ on line $h$ dominates the value of line $g$. For every line $g$ in the circuit, and every value $a \in\{0,1\}$, eight lines are selected randomly as the line $h$ that dominates $g$, and eight bridging faults are defined. Feedback bridging faults are avoided.

The set of bridging faults defined in this way is simulated under the one-detection test set, the improved one-detection test set, the ten-detection test set, and the improved ten-detection test set. The numbers of tests and bridging fault coverages are shown in Table 4 for circuits where all four test sets are available. 
Table 2. Experimental Results (ISCAS-89)

\begin{tabular}{|c|c|c|c|c|c|c|c|c|c|c|c|c|}
\hline circuit & pi & ts & tests & $\bmod$ & f.c. & 16det & undet & act & $\operatorname{targ}$ & 16act & miss & ntime \\
\hline s1423 & 91 & 1 & 38 & - & 99.076 & 8.422 & 14 & 14 & - & 12.000 & 1.601 & 1.05 \\
\hline s1423 & 91 & 1 & 38 & 11 & 99.076 & 8.550 & 14 & 14 & 13 & 12.000 & 1.548 & 65.05 \\
\hline s1423 & 91 & $1 \mathrm{G}$ & 41 & - & 99.076 & 8.718 & 14 & 14 & - & 12.214 & 1.588 & 1.00 \\
\hline s1423 & 91 & $1 \mathrm{G}$ & 41 & 14 & 99.076 & 8.865 & 14 & 14 & 13 & 12.214 & 1.539 & 61.58 \\
\hline s1423 & 91 & 10 & 269 & - & 99.076 & 15.376 & 14 & 14 & - & 16.000 & 1.143 & 1.05 \\
\hline $\mathrm{s} 1423$ & 91 & 10 & 269 & 3 & 99.076 & 15.538 & 14 & 14 & 2 & 16.000 & 1.089 & 586.77 \\
\hline s5378 & 214 & 1 & 111 & - & 99.131 & 11.442 & 40 & 30 & - & 13.300 & 1.252 & 1.01 \\
\hline s5378 & 214 & 1 & 111 & 5 & 99.131 & 11.691 & 40 & 30 & 25 & 13.433 & 1.188 & 227.93 \\
\hline s5378 & 214 & $1 \mathrm{G}$ & 117 & - & 99.131 & 11.656 & 40 & 30 & - & 13.467 & 1.234 & 1.01 \\
\hline s5378 & 214 & $1 \mathrm{G}$ & 117 & 5 & 99.131 & 11.922 & 40 & 30 & 24 & 13.600 & 1.174 & 242.12 \\
\hline s5378 & 214 & 10 & 992 & - & 99.131 & 15.512 & 40 & 30 & - & 15.967 & 1.043 & 1.03 \\
\hline s5378 & 214 & 10 & 992 & 883 & 99.131 & 15.525 & 40 & 30 & 11 & 16.000 & 1.000 & 227.88 \\
\hline s9234 & 247 & 1 & 143 & - & 93.475 & 9.280 & 452 & 446 & - & 15.265 & 1.890 & 1.00 \\
\hline s9234 & 247 & 1 & 143 & 3 & 93.475 & 9.554 & 452 & 446 & 359 & 15.271 & 1.845 & 294.72 \\
\hline s9234 & 247 & $1 \mathrm{G}$ & 214 & - & 93.475 & 10.379 & 452 & 446 & - & 15.343 & 1.614 & 1.01 \\
\hline s9234 & 247 & $1 \mathrm{G}$ & 214 & 0 & 93.475 & 10.678 & 452 & 446 & 319 & 15.350 & 1.570 & 444.94 \\
\hline s9234 & 247 & 10 & 1,132 & - & 93.475 & 15.145 & 452 & 447 & - & 15.767 & 1.107 & 1.01 \\
\hline s9234 & 247 & 10 & 1,132 & 42 & 93.475 & 15.298 & 452 & 447 & 150 & 15.781 & 1.032 & $2,225.25$ \\
\hline s13207 & 700 & 1 & 238 & - & 98.462 & 11.409 & 151 & 145 & - & 14.469 & 1.152 & 1.00 \\
\hline $\mathrm{s} 13207$ & 700 & 1 & 238 & 2 & 98.462 & 11.830 & 151 & 145 & 53 & 15.690 & 1.082 & 507.82 \\
\hline s13207 & 700 & $1 \mathrm{G}$ & 305 & - & 98.462 & 12.079 & 151 & 145 & - & 15.945 & 1.159 & 1.01 \\
\hline s13207 & 700 & $1 \mathrm{G}$ & 305 & 11 & 98.462 & 12.435 & 151 & 145 & 43 & 16.000 & 1.034 & 592.99 \\
\hline s13207 & 700 & 10 & 2,341 & - & 98.462 & 15.134 & 151 & 145 & - & 15.834 & 0.943 & 1.01 \\
\hline $\mathrm{s} 13207$ & 700 & 10 & 2,341 & 7 & 98.462 & 15.165 & 151 & 145 & 15 & 15.855 & 0.911 & $4,774.37$ \\
\hline s15850 & 611 & 1 & 118 & - & 96.682 & 11.183 & 389 & 365 & - & 14.227 & 1.560 & 1.00 \\
\hline s15850 & 611 & 1 & 118 & 0 & 96.682 & 11.597 & 389 & 365 & 196 & 14.293 & 1.359 & 271.19 \\
\hline s15850 & 611 & $1 \mathrm{G}$ & 302 & - & 96.682 & 13.284 & 389 & 365 & - & 15.918 & 1.344 & 1.01 \\
\hline $\mathrm{s} 15850$ & 611 & $1 \mathrm{G}$ & 302 & 1 & 96.682 & 13.623 & 389 & 365 & 106 & 15.918 & 1.104 & 624.61 \\
\hline s15850 & 611 & 10 & 983 & - & 96.682 & 15.441 & 389 & 365 & - & 15.449 & 1.203 & 1.01 \\
\hline $\mathrm{s} 15850$ & 611 & 10 & 983 & 4 & 96.682 & 15.504 & 389 & 365 & 84 & 15.449 & 1.098 & $2,094.26$ \\
\hline s35932 & 1,763 & 1 & 20 & - & 89.809 & 4.893 & 3,984 & 3,983 & - & 7.475 & 1.998 & 1.00 \\
\hline s35932 & 1,763 & 10 & 129 & - & 89.809 & 15.188 & 3,984 & 3,984 & - & 16.000 & 1.362 & 1.00 \\
\hline s35932 & 1,763 & 10 & 129 & 0 & 89.809 & 15.296 & 3,984 & 3,984 & 2,309 & 16.000 & 1.328 & 365.29 \\
\hline s38417 & 1,664 & 1 & 103 & - & 99.471 & 11.462 & 165 & 152 & - & 14.099 & 1.582 & 1.00 \\
\hline s38417 & 1,664 & 1 & 103 & 0 & 99.471 & 12.022 & 165 & 152 & 125 & 14.099 & 1.516 & 330.20 \\
\hline s38417 & 1,664 & 10 & 784 & - & 99.471 & 15.586 & 165 & 152 & - & 16.000 & 1.147 & 1.01 \\
\hline s38417 & 1,664 & 10 & 784 & 4 & 99.471 & 15.678 & 165 & 152 & 27 & 16.000 & 1.061 & $1,725.92$ \\
\hline s38584 & 1,464 & 1 & 142 & - & 95.852 & 11.038 & 1,506 & 1,363 & - & 11.014 & 1.526 & 1.00 \\
\hline s38584 & 1,464 & 1 & 142 & 1 & 95.852 & 11.518 & 1,506 & 1,363 & 1,029 & 11.066 & 1.501 & 335.06 \\
\hline s38584 & 1,464 & 10 & 1,191 & - & 95.852 & 15.676 & 1,506 & 1,363 & - & 15.996 & 1.444 & 1.00 \\
\hline s38584 & 1,464 & 10 & 1,191 & 181 & 95.852 & 15.770 & 1,506 & 1,363 & 796 & 15.996 & 1.442 & $2,175.88$ \\
\hline
\end{tabular}


Table 3. Experimental Results (ITC-99 and IWLS-05)

\begin{tabular}{|c|c|c|c|c|c|c|c|c|c|c|c|c|}
\hline circuit & pi & ts & tests & $\bmod$ & f.c. & 16det & undet & act & $\operatorname{targ}$ & 16act & miss & ntime \\
\hline b04 & 78 & 1 & 44 & - & 99.851 & 9.029 & 2 & 2 & - & 16.000 & 3.031 & 1.00 \\
\hline b04 & 78 & 1 & 44 & 3 & 99.851 & 9.421 & 2 & 2 & 2 & 16.000 & 2.562 & 95.75 \\
\hline b04 & 78 & $1 \mathrm{G}$ & 47 & - & 99.851 & 9.260 & 2 & 2 & - & 16.000 & 2.594 & 1.06 \\
\hline b04 & 78 & $1 \mathrm{G}$ & 47 & 10 & 99.851 & 9.622 & 2 & 2 & 2 & 16.000 & 2.219 & 85.83 \\
\hline b04 & 78 & 10 & 348 & - & 99.851 & 15.518 & 2 & 2 & - & 16.000 & 1.000 & 1.07 \\
\hline b05 & 36 & 1 & 61 & - & 95.209 & 8.671 & 87 & 74 & - & 13.743 & 2.226 & 1.02 \\
\hline b05 & 36 & 1 & 61 & 1 & 95.209 & 8.728 & 87 & 74 & 84 & 13.770 & 2.128 & 125.97 \\
\hline b05 & 36 & $1 \mathrm{G}$ & 83 & - & 95.209 & 9.961 & 87 & 74 & - & 13.946 & 1.948 & 1.02 \\
\hline b05 & 36 & $1 \mathrm{G}$ & 83 & 6 & 95.209 & 10.049 & 87 & 74 & 80 & 13.959 & 1.846 & 158.64 \\
\hline b05 & 36 & 10 & 514 & - & 95.209 & 15.548 & 87 & 74 & - & 16.000 & 1.329 & 1.07 \\
\hline b05 & 36 & 10 & 514 & 41 & 95.209 & 15.696 & 87 & 74 & 32 & 16.000 & 1.232 & $1,062.10$ \\
\hline$\overline{b 11}$ & 38 & 1 & 59 & - & 99.174 & 7.878 & 9 & 7 & - & 10.571 & 2.738 & 1.00 \\
\hline b11 & 38 & 1 & 59 & 22 & 99.174 & 8.032 & 9 & 7 & 9 & 10.571 & 2.729 & 78.54 \\
\hline$\overline{\mathrm{b} 11}$ & 38 & $1 \mathrm{G}$ & 68 & - & 99.174 & 8.312 & 9 & 7 & - & 11.714 & 2.411 & 1.00 \\
\hline b11 & 38 & $1 \mathrm{G}$ & 68 & 12 & 99.174 & 8.563 & 9 & 7 & 9 & 11.714 & 2.384 & 115.19 \\
\hline$\overline{b 11}$ & 38 & 10 & 572 & - & 99.174 & 14.912 & 9 & 7 & - & 16.000 & 1.589 & 1.07 \\
\hline b11 & 38 & 10 & 572 & 88 & 99.174 & 15.056 & 9 & 7 & 7 & 16.000 & 1.527 & $1,063.40$ \\
\hline b14 & 280 & 1 & 332 & - & 94.870 & 10.607 & 512 & 472 & - & 15.015 & 2.241 & 1.01 \\
\hline b14 & 280 & 1 & 332 & 2 & 94.880 & 10.746 & 511 & 471 & 373 & 15.025 & 2.068 & 704.99 \\
\hline b14 & 280 & $1 \mathrm{G}$ & 656 & - & 95.241 & 11.449 & 475 & 435 & - & 14.963 & 1.590 & 1.01 \\
\hline b14 & 280 & $1 \mathrm{G}$ & 656 & 6 & 95.291 & 11.659 & 470 & 430 & 248 & 14.965 & 1.440 & $1,360.53$ \\
\hline$\overline{b 14}$ & 280 & 10 & 3,058 & - & 94.870 & 15.490 & 512 & 463 & - & 15.577 & 1.397 & 1.02 \\
\hline b14 & 280 & 10 & 3,058 & 0 & 95.131 & 15.519 & 486 & 437 & 159 & 15.586 & 1.208 & $5,379.47$ \\
\hline$\overline{b 15}$ & 483 & 1 & 393 & - & 98.445 & 11.014 & 312 & 295 & - & 14.264 & 1.445 & 1.00 \\
\hline b15 & 483 & 1 & 393 & 27 & 98.445 & 11.546 & 312 & 296 & 187 & 14.412 & 1.405 & 723.99 \\
\hline b15 & 483 & $1 \mathrm{G}$ & 418 & - & 98.445 & 11.135 & 312 & 295 & - & 14.342 & 1.435 & 1.00 \\
\hline b15 & 483 & $1 G$ & 418 & 7 & 98.445 & 11.673 & 312 & 296 & 177 & 14.601 & 1.390 & 837.41 \\
\hline $\mathrm{b} 20$ & 527 & 1 & 302 & - & 94.056 & 11.115 & 1,342 & 1266 & - & 15.115 & 2.559 & 1.00 \\
\hline b20 & 527 & 1 & 302 & 0 & 94.056 & 11.264 & 1,342 & 1266 & 1,037 & 15.122 & 2.487 & 660.99 \\
\hline tv80 & 372 & 1 & 489 & - & 99.329 & 10.787 & 105 & 95 & - & 15.895 & 2.236 & 1.00 \\
\hline tv80 & 372 & 1 & 489 & 15 & 99.329 & 11.148 & 105 & 95 & 88 & 15.895 & 2.154 & 974.35 \\
\hline tv80 & 372 & 10 & 4,095 & - & 99.348 & 15.487 & 102 & 92 & - & 16.000 & 1.424 & 1.00 \\
\hline tv80 & 372 & 10 & 4,095 & 428 & 99.361 & 15.588 & 100 & 90 & 50 & 16.000 & 1.344 & $7,580.50$ \\
\hline
\end{tabular}

In most of the cases, the improved test set achieves a higher bridging fault coverage than the initial test set to which the improvement procedure is applied. This is consistent with the increase in the average number of detections reported in Tables 2 and 3. The improvement is not large, because the number of tests is not increased, and the initial bridging fault coverage is already high. Nevertheless, it provides further evidence to the improvement in the quality of the test set and its ability to detect defects.

\section{CONCLUDING REMARKS}

This article defined the concept of an incomplete test that is relevant when a target fault is undetectable. An incomplete test activates the fault, but fails to detect it because of one or more 


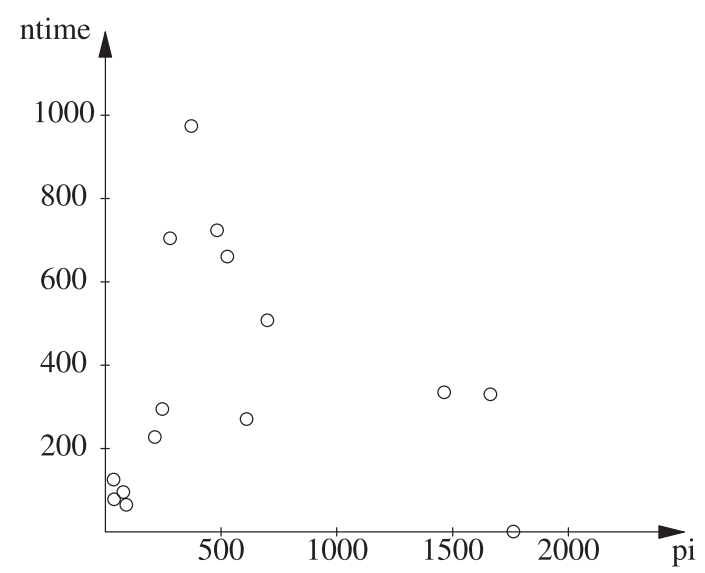

Fig. 5. Normalized runtime.

Table 4. Bridging Fault Coverage

\begin{tabular}{l|ccc|ccc}
\hline & \multicolumn{3}{|c|}{ 1-det } & \multicolumn{3}{c}{ 10-det } \\
circuit & tests & init & improv & tests & init & improv \\
\hline s1423 & 38 & 85.594 & 86.028 & 269 & 94.805 & 95.235 \\
s5378 & 111 & 90.099 & 90.449 & 992 & 97.560 & 97.575 \\
s9234 & 143 & 85.957 & 86.350 & 1,132 & 95.677 & 95.696 \\
s13207 & 238 & 88.667 & 89.227 & 2,341 & 97.375 & 97.318 \\
s15850 & 118 & 90.756 & 91.179 & 983 & 97.728 & 97.650 \\
s38417 & 103 & 93.698 & 94.260 & 784 & 99.449 & 99.486 \\
s38584 & 142 & 85.048 & 85.691 & 1,191 & 92.805 & 92.822 \\
\hline b05 & 61 & 83.733 & 83.876 & 514 & 93.702 & 93.980 \\
b11 & 59 & 76.108 & 76.293 & 572 & 82.375 & 82.925 \\
b14 & 332 & 82.923 & 83.102 & 3,058 & 87.916 & 88.057 \\
tv80 & 489 & 83.734 & 84.092 & 4,095 & 91.026 & 91.269 \\
\hline
\end{tabular}

assignments that are missing from the test. A smaller number of missing assignments makes it more likely that detectable defects, which should have been detected by a test for the undetectable fault, will be detected. Based on this concept, the article described a procedure that improves the quality of a test set in a way that fits with the rationale for computing an $n$-detection test set. For a constant $n \geq 1$, the procedure attempts to ensure that every undetectable fault has $n$ incomplete tests with the smallest possible numbers of missing assignments. The computation of missing assignments was performed in linear time by avoiding fault simulation and considering all the undetectable faults simultaneously. Experimental results were presented to demonstrate the extent to which a given test set can be improved without increasing the number of tests. Both one-detection and ten-detection test sets were considered, as well as one-detection test sets that are extended with tests for gate-exhaustive faults.

\section{REFERENCES}

[1] S. T. Zachariah and S. Chakravarty. 2000. A scalable and efficient methodology to extract two node bridges from large industrial circuits. In Proceedings of the International Test Conference. 750-759. 
[2] V. Krishnaswamy, A. B. Ma, and P. Vishakantaiah. 2001. A study of bridging defect probabilities on a pentium (TM) 4 CPU. In Proceedings of the International Test Conference. 688-695.

[3] I. Polian, S. Kundu, J.-M. Galliere, P. Engelke, M. Renovell, and B. Becker. 2005. Resistive bridge fault model evolution from conventional to ultra deep submicron technologies. In Proceedings of the VLSI Test Symposium. 343-348.

[4] S. K. Goel, N. Devta-Prasanna, and M. Ward. 2009. Comparing the effectiveness of deterministic bridge fault and multiple-detect stuck fault patterns for physical bridge defects: A simulation and silicon study. In Proceedings of the International Test Conference. 1-10.

[5] S. Khursheed, B. M. Al-Hashimi, and P. Harrod. 2009. Test cost reduction for multiple-voltage designs with bridge defects through gate-sizing. In Proceedings of the Design, Automation and Test in Europe Conference. 1349-1354.

[6] P. Engelke, B. Becker, M. Renovell, J. Schloeffel, B. Braitling, and I. Polian. 2009. SUPERB: Simulator utilizing parallel evaluation of resistive bridges. ACM Trans. Design Automat. 14, 4 (2009), Article 56.

[7] C.-H. Wu, S. J. Lee, and K.-J. Lee. 2016. Test and diagnosis pattern generation for dynamic bridging faults and transition delay faults. In Proceedings of the Asia and South Pacific Design Automation Conference. 755-760.

[8] M. Renovell and G. M. Cambon. 1992. Electrical analysis of floating-gate fault. IEEE Trans. Comput.-Aided Design 1450-1458.

[9] H. Konuk and F. J. Ferguson. 1996. An unexpected factor in testing for CMOS opens: The die surface. In Proceedings of the VLSI Test Symposium. 422-429.

[10] V. H. Champac and A. Zenteno. 2005. Detectability conditions for interconnect open defects. In Proceedings of the VLSI Test Symposium. 305-311.

[11] D. Arumi, R. Rodriguez-Montanes, and J. Figueras. 2005. Defective behaviours of resistive opens in interconnect lines. In Proceedings of the European Test Symposium. 28-33.

[12] R. Gomez, A. Giron, and V. Champac. 2005. Test of interconnect opens considering coupling signals. In Proceedings of the Defect and Fault Tolerant VLSI Systems Symposium. 247-255.

[13] H. Takahashi, Y. Higami, S. Kadoyama, T. Aikyo, Y. Takamatsu, K. Yamazaki, H. Yotsuyanagi, and M. Hashizume. 2007. Clues for modeling and diagnosing open faults with considering adjacent lines. In Proceedings of the Asian Test Symposium. 39-44.

[14] A. Sreedhar, A. Sanyal, and S. Kundu. 2008. On modeling and testing of lithography related open faults in nano-CMOS circuits. In Proceedings of the Design, Automation and Test in Europe Conference. 616-621.

[15] S. Spinner, I. Polian, P. Engelke, B. Becker, M. Keim, and W.-T. Cheng. 2008. Automatic test pattern generation for interconnect open defects. In Proceedings of the VLSI Test Symposium. 181-186.

[16] D. Erb, K. Scheibler, M. Sauer, and B. Becker. 2014. Efficient SMT-based ATPG for interconnect open defects. In Proceedings of the Design, Automation and Test in Europe Conference, Article 125.

[17] M. O. Simsir, A. Bhoj, and N. K. Jha. 2010. Fault modeling for FinFET circuits. In Proceedings of the International Symposium on Nanoscale Architecture. 41-46.

[18] J. Zha, X. Cui, and C. L. Lee. 2012. Modeling and testing of interference faults in the nano NAND flash memory. In Proceedings of the Design, Automation and Test in Europe Conference. 527-531.

[19] Y. Liu and Q. Xu. 2012. On modeling faults in FinFET logic circuits. In Proceedings of the International Test Conference. 1-9.

[20] H. G. Mohammadi, P.-E. Gaillardon, and G. De Micheli. 2015. Fault modeling in controllable polarity silicon nanowire circuits. In Proceedings of the Design, Automation and Test in Europe Conference. 453-458.

[21] F. Hapke and J. Schloeffel. 2012. Introduction to the defect-oriented cell-aware test methodology for significant reduction of DPPM rates. In Proceedings of the European Test Symposium. 1-6.

[22] F. Hapke, W. Redemund, A. Glowatz, J. Rajski, M. Reese, M. Hustava, M. Keim, J. Schloeffel, and A. Fast. 2014. Cellaware test. IEEE Trans. Comput.-Aided Design 33, 9 (2014), 1396-1409.

[23] F. Yang, S. Chakravarty, A. Gunda, N. Wu, and J. Ning. 2014. Silicon evaluation of cell-aware ATPG tests and small delay tests. In Proceedings of the Asian Test Symposium. 101-106.

[24] A. D. Singh. 2016. Cell aware and stuck-open tests. In Proceedings of the European Test Symposium. 1-6.

[25] E. J. McCluskey. 1993. Quality and single-stuck faults. In Proceedings of the International Test Conference. 597.

[26] R. D. Blanton and J. P. Hayes. 1997. Properties of the input pattern fault model. In Proceedings of the International Conference on Computer Design. 372-380.

[27] K. Y. Cho, S. Mitra, and E. J. McCluskey. 2005. Gate exhaustive testing. In Proceedings of the International Test Conference. Paper 31.3, 1-7.

[28] R. Guo, S. Mitra, E. Amyeen, J. Lee, S. Sivaraj, and S. Venkataraman. 2006. Evaluation of test metrics: Stuck-at, bridge coverage estimate and gate exhaustive. In Proceedings of the VLSI Test Symposium. 66-71.

[29] A. Jas, S. Natarajan, and S. Patil. 2007. The region-exhaustive fault model. In Proceedings of the Asian Test Symposium. 13-18. 
[30] B. Benware, C. Schuermyer, N. Tamarapalli, K.-H. Tsai, S. Ranganathan, R. Madge, J. Rajski, and P. Krishnamurthy. 2003. Impact of multiple-detect test patterns on product quality. In Proceedings of the International Test Conference. 1031-1040.

[31] S. Venkataraman, S. Sivaraj, E. Amyeen, S. Lee, A. Ojha, and R. Guo. 2004. An experimental study of $n$-detect scan ATPG patterns on a processor. In Proceedings of the VLSI Test Symposium. 23-28.

[32] H. Tang, G. Chen, S. M. Reddy, C. Wang, J. Rajski, and I. Pomeranz. 2005. Defect aware test patterns. In Proceedings of the Design Automation and Test in Europe Conference. 450-455.

[33] J. E. Nelson, J. G. Brown, R. Desineni, and R. D. Blanton. 2006. Multiple-detect ATPG based on physical neighborhoods. In Proceedings of the Design Automation Conference. 1099-1102.

[34] J. Geuzebroek, E. J. Marinissen, A. Majhi, A. Glowatz, and F. Hapke. 2007. Embedded multi-detect ATPG and its effect on the detection of unmodeled defects. In Proceedings of the International Test Conference. 1-10.

[35] K.-Y. Liao, S.-C. Hsu, and J. C.-M. Li. 2013. GPU-based n-detect transition fault ATPG. In Proceedings of the Design Automation Conference. Article 28.

[36] I. Pomeranz. 2017. A bridging fault model for line coverage in the presence of undetected transition faults. In Proceedings of the Design Automation and Test in Europe Conference.

[37] I. Pomeranz. 2018. Covering undetected transition fault sites with optimistic unspecified transition faults under multicycle tests. In Proceedings of the European Test Symposium.

Received September 2018; revised November 2018; accepted December 2018 